Check for updates

Cite this: RSC Adv., 2019, 9, 11774

Received 4th March 2019

Accepted 1st April 2019

DOI: $10.1039 / \mathrm{c} 9 \mathrm{ra01626 \textrm {k }}$

rsc.li/rsc-advances

\section{Study on the identification of Pinelliae rhizoma and Pinelliae pedatisectae rhizoma based on the characteristic component triglochinic acid}

\author{
Yong Jing, ${ }^{a}$ Yueyue Lai, ${ }^{a}$ Hui Chen, ${ }^{a}$ Min Li, (D) *a Juan Zhou ${ }^{b}$ and Zelun Lan ${ }^{c}$
}

For the first time, a monomeric compound, triglochinic acid, has been isolated from the tubers of Pinellia pedatisecta Schott with its structure analyzed using NMR. Its molecular formula is $\mathrm{C}_{7} \mathrm{H}_{8} \mathrm{O}_{6}$, with the chemical name (2E)-but-2-ene-1,2,4-tricarboxylic acid. Through HPLC-DAD and HPLC-MS analysis studies, it has concluded that Pinelliae rhizoma does not contain triglochinic acid, while Pinelliae pedatisectae rhizoma does. This key observation was used as a characteristic component to distinguish these two herbs. We analyzed 39 batches of Pinelliae rhizoma collected in herbal medicine market, among which triglochinic acid was detected in 27 batches, resulting in a adulteration ratio of Pinelliae rhizoma reaching $69.2 \%$. Our method demonstrates great potential for authenticating the products, thus ensuring the quality of Pinelliae rhizoma.

\section{Introduction}

Pinelliae rhizoma is the dry tubers of Pinellia ternata (Thunb.) Breit. ${ }^{1}$ It was documented as Diwen or Shuiyu in Shennong's Materia Medica (Shen Nong Ben Cao Jing), and other medical classics afterwards. During ancient times, counterfeit or fake products of Pinelliae rhizoma existed, such as Arisaema ringens (Thunb.) Schott in Tang Ben Cao by Su Gong and Pinellia pedatisecta Schott in Tu Jing Ben Cao. In modern times, Pinelliae rhizoma in rural areas was replaced by herbs of the same genus like Typhonii flagelliformis and Arisaema heterophyllum Blume according to Modern Chinese Materia Medica. ${ }^{2}$ Recently, Pinelliae rhizoma was discovered to be mixed with a large amount of Pinelliae pedatisectae rhizoma in the market, which greatly affected the quality of the herbs. Pinelliae pedatisectae rhizoma, also known as the palm leaf Pinellia and South Star, is the dry tubers of Pinellia pedatisecta Schott, documented in the Chinese Materia Medica Standards of Shandong, Hubei and Jiangsu, ${ }^{3-5}$ as well as in the Dictionary of Traditional Chinese Medicine ${ }^{6}$ and Chinese Flora. ${ }^{7}$

Pinelliae rhizoma and Pinelliae pedatisectae rhizoma are both of the Pinellia genus, which can easily be identified by the leaves and flowers. ${ }^{7}$ However, their medicinal materials are extremely similar. Pinelliae rhizoma is spheroidal in shape, while Pinelliae pedatisectae rhizoma is also spheroidal with several small bulbs alongside. If the small bulbs of Pinelliae pedatisectae rhizoma are not yet formed or

${ }^{a}$ Key Laboratory of Standardization of Chinese Herbal Medicine, State Key Laboratory Breeding Base of Systematic Research, Development and Utilization of Chinese Medicine Resources, Ministry of Education, College of Pharmacy, Chengdu University of Traditional Chinese Medicine, Chengdu 611137, China. E-mail: limin@cdutcm.edu.cn; Tel: +86-13980038316

${ }^{b}$ Sichuan Institute for Food and Drug Control, Chengdu 611731, China 'Sichuan Neautus Traditional Chinese Medicine Co., Ltd., Chengdu 611731, China removed during processing, the herbs will be very similar to Pinelliae rhizoma, which makes them difficult to distinguish. Pinelliae rhizoma enjoys a wide range of clinical applications, being the raw material in 489 patent traditional Chinese medicine ${ }^{8}$ and in 3029 prescriptions. ${ }^{9}$ The growing period of Pinellia ternata is $4-5$ months with high cost and low yield, and its market price is 85120 yuan per kilogram. On the other hand, the clinical application of Pinelliae pedatisectae rhizoma is limited, only used in a few prescriptions. Its lifespan is shorter, about 3-4 months with lower cost, higher yield and the price is $40-50$ yuan per kilogram. Therefore, the interests drive people to incorporate Pinelliae pedatisectae rhizoma into Pinelliae rhizoma.

Nowadays, the identifications of Pinelliae rhizoma, Typhonii flagelliformis rhizoma and Arisaematis rhizoma are mostly based on morphology identification, thin layer chromatography identification, and fingerprint identification. ${ }^{\mathbf{1 0 - 1 2}}$ However, the identification studies on Pinelliae rhizoma and Pinelliae pedatisectae rhizoma are very limited. In particular, it is hard to identify the incorporation of Pinelliae pedatisectae rhizoma in Pinelliae rhizoma. From 2016 to 2017, we collected Pinelliae rhizoma from several herbal medicine markets: Hehuachi, Anguo, Bozhou, Qingping, Yulin, etc. However, more than 60\% of the samples were counterfeit. This situation urges us to establish an effective and rapid analytical method that can detect fake herbs and improve the quality control of Pinelliae rhizoma, meanwhile laying the foundation of a standard herbal medicine market.

\section{Material}

\subsection{Equipment}

Agilent 1200 high performance liquid chromatography (with DAD detector), Agilent 1290/6460 LC/MS, Agilent ZORBAX 
Eclipse Plus $\mathrm{C}_{18}$ column $(4.6 \times 250 \mathrm{~mm}, 5 \mu \mathrm{m})$, Agilent ZORBAX Eclipse Plus $\mathrm{C}_{18}$ column $(2.1 \times 50 \mathrm{~mm}, 1.8 \mu \mathrm{m}), \mathrm{XB}^{-\mathrm{C}_{18}}$ column $(80 \times 250 \mathrm{~mm}, 10 \mu \mathrm{m}), 3-30 \mathrm{~K}$ centrifuge (Sigma, Germany), KQ-500DE CNC ultrasonic cleaner (Kunshan Ultrasonic Instrument Co., Ltd.); ULUP-IV-10T Youpu series ultrapure water (Chengdu Ultrapure Technology Co., Ltd.), SQP electronic balance (Sartorious Scientific Instrument Beijing Co., Ltd.).

\subsection{Reagents}

Triglochinic acid reference substance $(96.68 \%$ purity, batch number 20170201), purchased from Chengdu Push BioTechnology Co., Ltd. Phosphoric acid (Chengdu Kelon Chemical Reagent Factory), formic acid (Tianjin Kemiou Chemical Reagent Co., Ltd.) and acetonitrile (Thermo Fisher Scientific (China)) are chromatographically pure, and the water is ultrapure.

\subsection{Medicinal materials}

From Sichuan, Gansu, Guizhou, Hebei, Shanxi, Chongqing and other provinces and cities, we collected 28 batches of Pinelliae rhizoma. Also from Hebei, Heilongjiang, Gansu, Sichuan and other places, we collected 16 batches of Pinelliae pedatisectae rhizoma. All the materials were determined by Professor Min Li from College of Pharmacy, Chengdu University of Traditional Chinese Medicine. Information of these samples is shown in Table 1. Photos of Pinelliae rhizoma and Pinelliae pedatisectae are shown in Fig. 1.

In addition, 39 batches of commercial Pinelliae rhizoma were collected from the herbal medicine markets of Hehuachi Chengdu, Anguo Hebei, Yinzhou Anhui, Qingping Guangdong, and Yulin Guangxi. Information of the commercial Pinelliae rhizoma samples is shown in Table 2.

\section{Methods and results}

After a systematic study on the chemical components of Pinelliae rhizoma and Pinelliae pedatisectae rhizoma, we discovered a characteristic peak of Pinelliae pedatisectae rhizoma in the HPLC chromatogram of organic acid part, distinguishable from that of Pinelliae rhizoma.

\subsection{Extraction, separation and purification of the characteristic component}

Take the powder of Pinelliae pedatisectae rhizoma (after passing the no. 4 sieve), and add 10 times the amount of water. Sonicate the sample for 3 times, 1 hour each time. Then, combine the extracts and add phosphoric acid, $1 \%$ of the extract volume. Shake and add an equal volume of ethyl acetate for extracting 3 times. Recover the ethyl acetate to obtain the concentrated liquid before dilute it with water. Pass through the $\mathrm{XB}^{-\mathrm{C}_{18}}(80 \times$ $250 \mathrm{~mm}, 10 \mu \mathrm{m}$ ) preparative column at flow rate of $140 \mathrm{~mL}$ per minute, using $5 \%$ acetonitrile (containing $0.2 \%$ phosphoric acid solution) as the mobile phase, collect the eluent from 19.5 to 22.6 minutes. Then, recover the solvent and change the mobile phase to $5 \%$ acetonitrile (containing $0.1 \%$ formic acid solution). Use the column again and collect the eluate from 17.4
Table 1 Market Information of the samples

\begin{tabular}{|c|c|c|}
\hline ID & Sample & Origin \\
\hline BX1 & Pinelliae rhizoma & Muba, Nanchong, Sichuan \\
\hline BX2 & Pinelliae rhizoma & Muba, Nanchong, Sichuan \\
\hline BX3 & Pinelliae rhizoma & Jinchuan, Aba, Sihuan \\
\hline BX4 & Pinelliae rhizoma & Pengxi, Suining, Sichuan \\
\hline BX5 & Pinelliae rhizoma & Wenjiang, Chengdu, Sichuan \\
\hline BX6 & Pinelliae rhizoma & Nanjiang, Bazhong, Sichuan \\
\hline BX7 & Pinelliae rhizoma & Mianning, Liangshan, Sichuan \\
\hline BX8 & Pinelliae rhizoma & Mianning, Liangshan, Sichuan \\
\hline BX9 & Pinelliae rhizoma & Hongjiang, Suining, Sichuan \\
\hline BX10 & Pinelliae rhizoma & Changle, Nanchong, Sichuan \\
\hline BX11 & Pinelliae rhizoma & Shayang, Jinmen, Hubei \\
\hline BX12 & Pinelliae rhizoma & Xihe, Longnan, Gansu \\
\hline BX13 & Pinelliae rhizoma & Li, Longnan, Gansu \\
\hline BX14 & Pinelliae rhizoma & Qingshui, Tianshui, Gansu \\
\hline BX15 & Pinelliae rhizoma & Xihe, Longnan, Gansu \\
\hline BX16 & Pinelliae rhizoma & Qingshui, Tianshui, Gansu \\
\hline BX17 & Pinelliae rhizoma & Weining, Guizhou \\
\hline BX18 & Pinelliae rhizoma & Dafang, Guizhou \\
\hline BX19 & Pinelliae rhizoma & Hechuan, Chongqing \\
\hline BX20 & Pinelliae rhizoma & Shenze, Shijiazhuang, Hebei \\
\hline BX21 & Pinelliae rhizoma & Shenze, Shijiazhuang, Hebei \\
\hline BX22 & Pinelliae rhizoma & Shenze, Shijiazhuang, Hebei \\
\hline BX23 & Pinelliae rhizoma & Houma, Shanxi \\
\hline BX24 & Pinelliae rhizoma & Houma, Shanxi \\
\hline BX25 & Pinelliae rhizoma & Houma, Shanxi \\
\hline BX26 & Pinelliae rhizoma & Weining, Guizhou \\
\hline BX27 & Pinelliae rhizoma & Hezhang, Guizhou \\
\hline $\mathrm{BX} 28$ & Pinelliae rhizoma & Hezhang, Guizhou \\
\hline HZ1 & Pinelliae pedatisectae rhizoma & Qizhou, Anguo, Hebei \\
\hline $\mathrm{HZ2}$ & Pinelliae pedatisectae rhizoma & Zhengzhang, Anguo, Hebei \\
\hline $\mathrm{HZ3}$ & Pinelliae pedatisectae rhizoma & Zhengzhang, Anguo, Hebei \\
\hline $\mathrm{HZ4}$ & Pinelliae pedatisectae rhizoma & Xifuluo, Anguo, Hebei \\
\hline HZ5 & Pinelliae pedatisectae rhizoma & Qizhou, Anguo, Hebei \\
\hline HZ6 & Pinelliae pedatisectae rhizoma & Qizhou, Anguo, Hebei \\
\hline $\mathrm{HZ7}$ & Pinelliae pedatisectae rhizoma & Tongnan, Nehe, Heilongjia \\
\hline $\mathrm{HZ8}$ & Pinelliae pedatisectae rhizoma & Anguo, Hebei \\
\hline HZ9 & Pinelliae pedatisectae rhizoma & Anguo, Hebei \\
\hline HZ10 & Pinelliae pedatisectae rhizoma & Anguo, Hebei \\
\hline HZ11 & Pinelliae pedatisectae rhizoma & Tongnan, Nehe, Heilongjia \\
\hline HZ12 & Pinelliae pedatisectae rhizoma & Anguo, Hebei \\
\hline HZ13 & Pinelliae pedatisectae rhizoma & Muba, Nanchong, Sichuan \\
\hline HZ14 & Pinelliae pedatisectae rhizoma & Xihe, Longnan, Gansu \\
\hline HZ15 & Pinelliae pedatisectae rhizoma & Wenjiang, Chengdu, Sichuan \\
\hline HZ16 & Pinelliae pedatisectae rhizoma & $\mathrm{Li}$, Longnan, Gansu \\
\hline
\end{tabular}

to 20.3 minutes. Finally, lyophilize the eluate under reduced pressure to obtain the white compound in solid form.

\subsection{Structural identification of the characteristic component}

We used NMR to analyze the structure of the white compound. The data was listed in Table $3 .{ }^{1} \mathrm{H}-\mathrm{NMR}\left(600 \mathrm{MHz}, \mathrm{MeOD}-d_{4}\right)$ showed that the compound has five hydrogen signals: a double bond hydrogen signal $\delta_{\mathrm{H}} 7.13(1 \mathrm{H}, \mathrm{t}, J=7.2 \mathrm{~Hz})$ in the low field, two sets of hydrogen signals $\delta_{\mathrm{H}} 3.35(2 \mathrm{H}, \mathrm{s}), 3.27(2 \mathrm{H}, \mathrm{d}, J=7.2$ $\mathrm{Hz}$ ) in the high field. Correspondingly, 13C-NMR showed 7 carbon signals: 3 carbonyl carbon signals $\delta_{\mathrm{C}} 172.9,172.3,168.5$, a double bond signal $\delta_{\mathrm{C}} 136.9,128.4$ in the low field, $\delta_{\mathrm{C}} 33.3$, 31.7 in the high field. Combined with DEPT $135^{\circ}, \delta_{\mathrm{C}} 136.9$ is a methine, suggesting the double bond is trisubstituted, while 


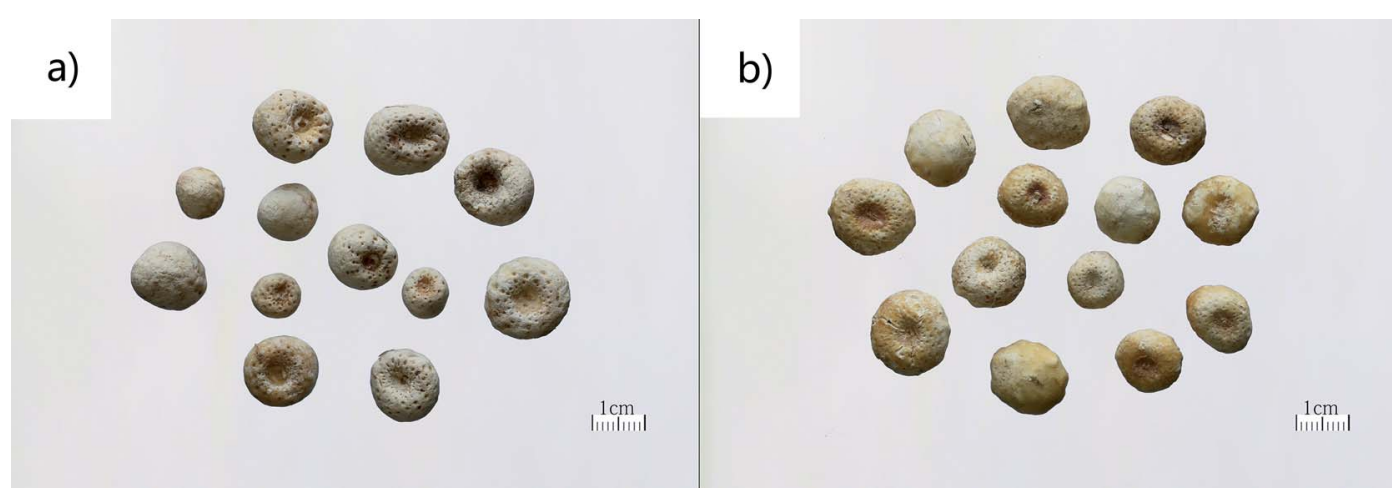

Fig. 1 Photos of Pinelliae rhizoma (a) and Pinelliae pedatisectae rhizoma (b).

$\delta_{\mathrm{C}} 33.3,31.7$ is methylene. HSQC carries out hydrocarbon partial assignment: $\delta_{\mathrm{C}} 136.9\left(\delta_{\mathrm{H}} 7.13\right), \delta_{\mathrm{C}} 33.3\left(\delta_{\mathrm{H}} 3.27\right), \delta_{\mathrm{C}} 31.7$ $\left(\delta_{\mathrm{H}} 3.35\right)$, and the link order of carbon is determined by HMBC correlation: $\delta_{\mathrm{H}} 7.13\left(\delta_{\mathrm{C}} 136.9, \mathrm{C}-3\right)$ is related to $\delta_{\mathrm{C}} 172.3(\mathrm{C}-1)$,

Table 2 Market information of the commercial herb samples

\begin{tabular}{|c|c|c|}
\hline ID & Sample & Market \\
\hline SSBX1 & Commercial Pinelliae rhizoma & Bozhou, Anhui \\
\hline SSBX2 & Commercial Pinelliae rhizoma & Bozhou, Anhui \\
\hline SSBX3 & Commercial Pinelliae rhizoma & Yulin, Guanxi \\
\hline SSBX4 & Commercial Pinelliae rhizoma & Hehuachi, Chegndu \\
\hline SSBX5 & Commercial Pinelliae rhizoma & Hehuachi, Chegndu \\
\hline SSBX6 & Commercial Pinelliae rhizoma & Hehuachi, Chegndu \\
\hline SSBX7 & Commercial Pinelliae rhizoma & Hehuachi, Chegndu \\
\hline SSBX8 & Commercial Pinelliae rhizoma & Hehuachi, Chegndu \\
\hline SSBX9 & Commercial Pinelliae rhizoma & Hehuachi, Chegndu \\
\hline SSBX10 & Commercial Pinelliae rhizoma & Hehuachi, Chegndu \\
\hline SSBX11 & Commercial Pinelliae rhizoma & Hehuachi, Chegndu \\
\hline SSBX12 & Commercial Pinelliae rhizoma & Hehuachi, Chegndu \\
\hline SSBX13 & Commercial Pinelliae rhizoma & Hehuachi, Chegndu \\
\hline SSBX14 & Commercial Pinelliae rhizoma & Hehuachi, Chegndu \\
\hline SSBX15 & Commercial Pinelliae rhizoma & Anguo, Hebei \\
\hline SSBX16 & Commercial Pinelliae rhizoma & Anguo, Hebei \\
\hline SSBX17 & Commercial Pinelliae rhizoma & Anguo, Hebei \\
\hline SSBX18 & Commercial Pinelliae rhizoma & Anguo, Hebei \\
\hline SSBX19 & Commercial Pinelliae rhizoma & Anguo, Hebei \\
\hline SSBX20 & Commercial Pinelliae rhizoma & Anguo, Hebei \\
\hline SSBX21 & Commercial Pinelliae rhizoma & Anguo, Hebei \\
\hline SSBX22 & Commercial Pinelliae rhizoma & Bozhou, Anhui \\
\hline SSBX23 & Commercial Pinelliae rhizoma & Bozhou, Anhui \\
\hline SSBX24 & Commercial Pinelliae rhizoma & Bozhou, Anhui \\
\hline SSBX25 & Commercial Pinelliae rhizoma & Bozhou, Anhui \\
\hline SSBX26 & Commercial Pinelliae rhizoma & Bozhou, Anhui \\
\hline SSBX27 & Commercial Pinelliae rhizoma & Bozhou, Anhui \\
\hline SSBX28 & Commercial Pinelliae rhizoma & Zhejiang \\
\hline SSBX29 & Commercial Pinelliae rhizoma & Qingping, Guangdong \\
\hline SSBX30 & Commercial Pinelliae rhizoma & Qingping, Guangdong \\
\hline SSBX31 & Commercial Pinelliae rhizoma & Qingping, Guangdong \\
\hline SSBX32 & Commercial Pinelliae rhizoma & Qingping, Guangdong \\
\hline SSBX33 & Commercial Pinelliae rhizoma & Qingping, Guangdong \\
\hline SSBX34 & Commercial Pinelliae rhizoma & Qingping, Guangdong \\
\hline SSBX35 & Commercial Pinelliae rhizoma & Anguo, Hebei \\
\hline SSBX36 & Commercial Pinelliae rhizoma & Anguo, Hebei \\
\hline SSBX37 & Commercial Pinelliae rhizoma & Anguo, Hebei \\
\hline SSBX38 & Commercial Pinelliae rhizoma & Anguo, Hebei \\
\hline SSBX39 & Commercial Pinelliae rhizoma & Anguo, Hebei \\
\hline
\end{tabular}

$168.5(\mathrm{C}-5), 128.4(\mathrm{C}-4)$, and $31.7\left(\mathrm{C}-1^{\prime}\right) . \delta_{\mathrm{H}} 3.27\left(\delta_{\mathrm{C}} 33.3, \mathrm{C}-2\right)$ is related to $\delta_{\mathrm{C}} 172.3(\mathrm{C}-1), 136.9(\mathrm{C}-3)$, and $128.4(\mathrm{C}-4) . \delta_{\mathrm{H}}$ $3.35\left(\delta_{\mathrm{C}} 31.7, \mathrm{C}-1^{\prime}\right)$ is related to $\mathrm{C} 172.9\left(\mathrm{C}-2^{\prime}\right), 168.5(\mathrm{C}-5), 136.9$ (C-3), and $128.4(\mathrm{C}-4)$.

The nuclear magnetic data of this compound is generally consistent with that of triglochinic acid as reported in the literature. ${ }^{13}$ Therefore, this compound was identified as triglochinic acid, a white powdery solid, soluble in water and methanol, with the molecular formula $\mathrm{C}_{7} \mathrm{H}_{8} \mathrm{O}_{6}$. The chemical name is $(2 E)$-but-2-ene-1,2,4-tricarboxylic acid, CAS number: $31795-12-7$. Its relative molecular mass is 188.13 , boiling point $(557.3 \pm 50){ }^{\circ} \mathrm{C}$. The structure of triglochinic acid is depicted in Fig. 2.

\subsection{Selection of the detection wavelength}

With full-wavelength ultraviolet scanning, the maximum absorption wavelength of triglochinic acid is $210 \mathrm{~nm}$. Therefore, $210 \mathrm{~nm}$ was selected as the detection wavelength. The spectrum is shown in Fig. 3.

\subsection{Chromatographic conditions}

The column was an Agilent ZORBAX Eclipse Plus $\mathrm{C}_{18}$ column $(4.6 \times 250 \mathrm{~mm}, 5 \mu \mathrm{m})$. Acetonitrile (A) and $0.1 \%$ phosphoric acid solution (B) were applied as the mobile phase, using gradient elution (0-40 min, $1 \% \mathrm{~A} ; 40-45 \mathrm{~min}, 1-10 \% \mathrm{~A} ; 45-$ $50 \mathrm{~min}, 10-1 \% \mathrm{~A} ; 50-60 \mathrm{~min}, 1 \% \mathrm{~A} ; 30-35 \mathrm{~min}, 10 \% \mathrm{~A}$; $35-$ $40 \mathrm{~min}, 10-0 \% \mathrm{~A} ; 40-50 \mathrm{~min}, 0 \% \mathrm{~A})$ at the flow rate of $0.8 \mathrm{~mL}$ per minute, $25{ }^{\circ} \mathrm{C}$ column temperature, and $210 \mathrm{~nm}$ detection

Table 3 Carbon and hydrogen assignments of the compound $\left(\mathrm{MeOD}-d_{4}, \delta \mathrm{ppm}, \mathrm{J}=\mathrm{Hz}\right)$

\begin{tabular}{lcl}
\hline No. & $\delta_{\mathrm{C}}(150 \mathrm{MHz})$ & $\delta_{\mathrm{H}}(600 \mathrm{MHz})$ \\
\hline 1 & 172.3 & \\
2 & 33.3 & $3.27 \mathrm{~d}, J=7.2$ \\
3 & 136.9 & $7.13 \mathrm{t}, J=7.2$ \\
4 & 128.4 & \\
5 & 168.5 & $3.35 \mathrm{~s}$ \\
$1^{\prime}$ & 31.7 & \\
$2^{\prime}$ & 172.9 &
\end{tabular}




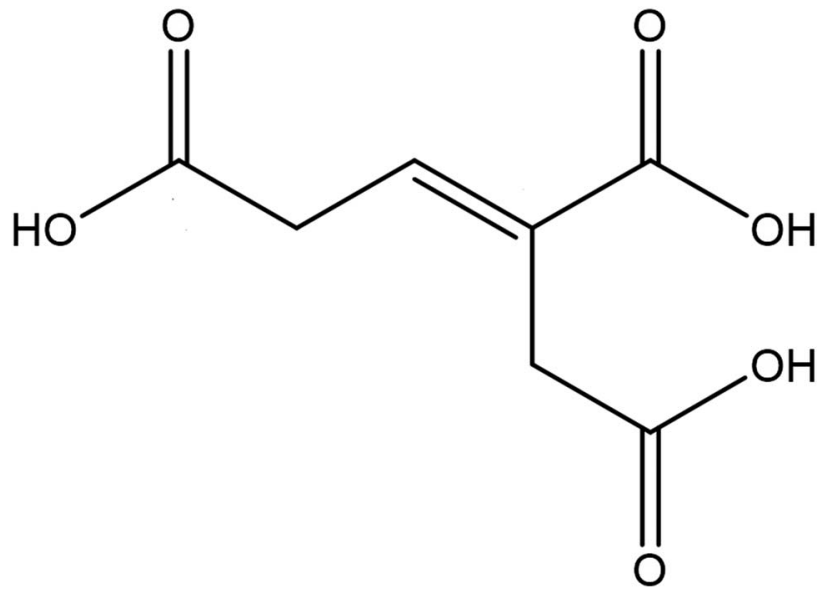

Fig. 2 Structural formula of triglochinic acid.

wavelength. The injection volume was $10 \mu \mathrm{L}$. The result is shown in Fig. 4.

\subsection{Solution preparation}

Preparation of the reference solution: accurately weigh an appropriate amount of triglochinic acid. Add water to make the reference solution with a concentration of $0.25 \mu \mathrm{g} \mathrm{mL}{ }^{-1}$.

Preparation of the test solution: accurately weigh about $1 \mathrm{~g}$ of the sample powder (after passing through the no. 4 sieve) and place it in an Erlenmeyer flask with $20 \mathrm{~mL}$ of water. Weigh before and after 45 minutes of sonication $(250 \mathrm{~W}, 40 \mathrm{kHz})$, add the lost weight with water. Filter the solution and transfer $10 \mathrm{~mL}$ of the filtrate to a $50 \mathrm{~mL}$ centrifuge tube. Add $0.1 \mathrm{~mL}$ phosphoric acid and shake, then add $20 \mathrm{~mL}$ ethyl acetate and shake. Centrifuge $(5000 \mathrm{rpm})$ the solution, and aspirate the upper liquid ethyl acetate. Extract acid solution with ethyl acetate for three more times, $20 \mathrm{~mL}$ each time. Then combine the ethyl acetate solution and evaporate the solvent to dry under reduced pressure. Add $2 \mathrm{~mL}$ acetonitrile- $0.1 \%$ phosphoric acid (1:99) into the residue to dissolve. Finally, filter it through a microporous membrane $(0.45 \mu \mathrm{m})$ and obtain the filtrate.

\subsection{Determination method}

Inject $10 \mu \mathrm{L}$ of the reference solution and test solution into HPLC, record the chromatogram.

\subsection{Methodology}

3.7.1 Precision of the experiment. Take the reference solution and the test solution, and inject the sample for 6 times according to the chromatographic conditions (3.4). Then, calculate the peak area of triglochinic acid in both reference and test solution. The RSD is less than $2 \%$, indicating that the instrument is precise.

3.7.2 Repeatability test. Take 6 samples of the same batch of Pinelliae pedatisectae rhizoma, and accurately weigh $1 \mathrm{~g}$. Follow the method in 3.5 to prepare the test solution and the chromatographic conditions in 3.4 for tests. The RSD of peak area of triglochinic acid was $0.57 \%$, indicating that this method is repetitive.

3.7.3 Stability test. Take the same test solution, and store it at room temperature. Follow the chromatographic method in 3.4 to test at $0 \mathrm{~h}, 4 \mathrm{~h}, 8 \mathrm{~h}, 12 \mathrm{~h}, 18 \mathrm{~h}$ and $24 \mathrm{~h}$. The results showed that the RSD of peak area of triglochinic acid from test solution was $0.92 \%$, indicating that the sample solution was relatively stable within 24 hours.

3.7.4 Detection limit. Take the reference solution, and dilute it to $0.10 \mu \mathrm{g} \mathrm{mL} \mathrm{m}^{-1}$ with water. Accurately inject $10 \mu \mathrm{L}$ into the HPLC, and calculate the signal-to-noise ratio $(\mathrm{S} / \mathrm{N}=3$ as the detection limit). The detection limit was $545.5 \mu \mathrm{g} \mathrm{kg}^{-1}$ (Table 4).

\subsection{Sample tests}

28 batches of Pinelliae rhizoma, 16 batches of Pinelliae pedatisectae rhizoma and 39 batches of commercial medicinal materials were prepared according to the method in 3.5, and determined following the chromatographic conditions in 3.4. If there is a peak consistent with the retention time of triglochinic acid, and the absorption spectrum of the corresponding chromatographic peak is the same within the wavelength range of 190-400 $\mathrm{nm}$ by the diode-array detector, the sample will be identified as Pinelliae pedatisectae rhizoma. The results were shown in Table 5.

The results showed that triglochinic acid was not detected in the 28 batches of Pinelliae rhizoma, while detected in all the 16 batches of Pinelliae pedatisectae rhizoma. However, triglochinic acid were detected in 27 of the 39 batches of the commercial samples. Therefore, the rate of fake products of Pinelliae rhizoma was $69.2 \%$.

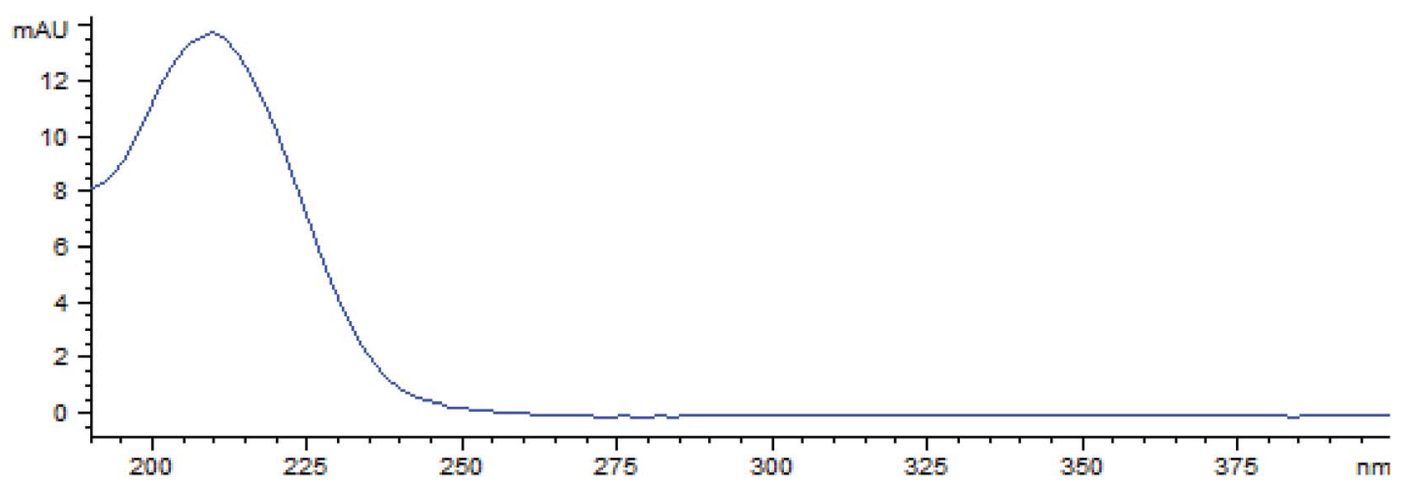

Fig. 3 Ultraviolet spectrum of triglochinic acid. 
Table 4 Detection limit ${ }^{a}$

\begin{tabular}{lllll}
\hline Component & $\begin{array}{l}\text { Concentration of the } \\
\text { reference solution }\left(\mu \mathrm{g} \mathrm{mL} \mathrm{mL}^{-1}\right)\end{array}$ & $\begin{array}{l}\text { Signal-to-noise } \\
\text { ratio S/N }\end{array}$ & $\begin{array}{l}\text { Dilution ration of } \\
\text { the test solution }\end{array}$ & $\begin{array}{l}\text { Sample } \\
\mathrm{amount}(\mathrm{g})\end{array}$ \\
\hline Triglochinic acid & 0.10 & 2.2 & 4 & $\begin{array}{l}\text { Detection limit } \\
\left(\mu \mathrm{g} \mathrm{kg} \mathrm{kg}^{-1}\right)\end{array}$ \\
\hline
\end{tabular}

${ }^{a}$ NB: detection limit $\left(\mu \mathrm{g} \mathrm{kg}^{-1}\right)=$ concentration of reference solution $\times$ injection volume of reference solution $\times 3 \times$ dilution ratio of test solution/ signal-to-noise ratio/sample amount/sample injection volume.

Table 5 Triglochinic acid test results in each sample ${ }^{a}$

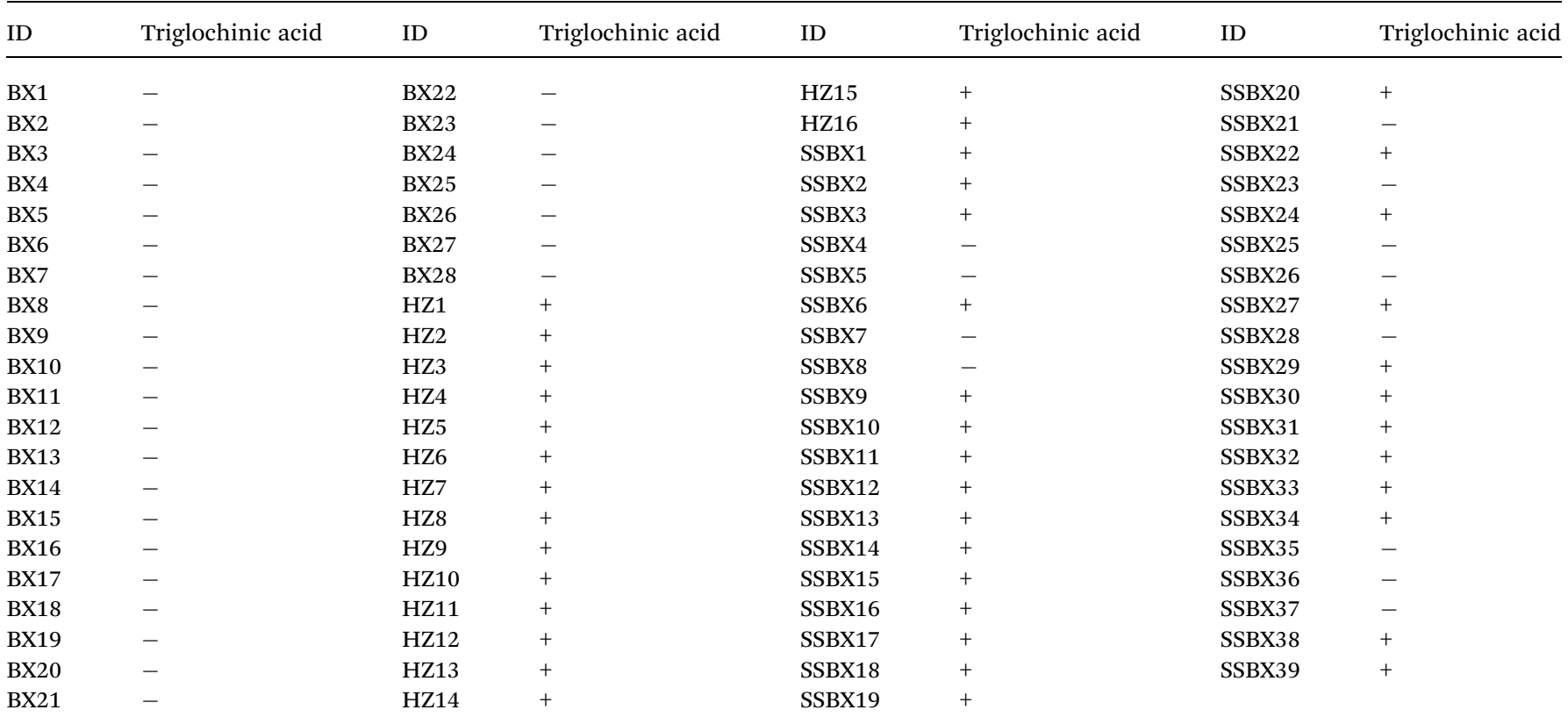

\footnotetext{
${ }^{a}$ NB: “+” for triglochinic acid detected, "-" for not.
} 
Table 6 Multi-reaction detection of ion ratios for triglochinic acid

\begin{tabular}{lll}
\hline Parent ion & Daughter ion & Collision energy \\
\hline 187 & 143 & 5 \\
187 & 99 & 9
\end{tabular}

Table 7 lon ratio of triglochinic acid in reaction monitoring

\begin{tabular}{lll}
\hline Sample name & Set ratio & Actual ratio \\
\hline Triglochinic acid & 96.37 & 96.37 \\
Pinelliae pedatisectae rhizoma & 96.37 & 93.38 \\
Pinelliae rhizoma & 96.37 & -
\end{tabular}

\subsection{HPLC-MS verification of triglochinic acid in the samples}

3.9.1 Liquid chromatographic conditions. The Agilent 1290-6460 LC/MS was used with Agilent ZORBAX Eclipse Plus $\mathrm{C}_{18}$ column $(2.1 \times 50 \mathrm{~mm}, 1.8 \mu \mathrm{m})$ and the mobile phase of methanol-0.02\% ammonia solution (5:95) at $0.15 \mathrm{~mL} \mathrm{~min}^{-1}$ flow rate, $45{ }^{\circ} \mathrm{C}$ column temperature. The injection volume was $2 \mu \mathrm{L}$.

3.9.2 Mass spectrometry conditions. Multi-reaction monitoring (MRM) was performed with a mass spectrometer detector, in electrospray negative ion mode $\left(\mathrm{ESI}^{-}\right)$at 3500 capillary voltage. Flow rate of dryer was $9 \mathrm{~L}$ per minute, temperature of dryer was $280{ }^{\circ} \mathrm{C}, 40$ psi Nebulizer, and $0 \mathrm{~V}$ Fragmentor (secondary). The parameters of triglochinic acid were shown in Table 6.

3.9.3 Preparation of the solution. In the preparation of the test solution, the acidifying reagent phosphoric acid was replaced by formic acid, while the remaining was as same as in 3.5.

3.9.4 Results verification. The multi-reaction detection of ion ratios for triglochinic acid, Pinelliae rhizoma, and Pinelliae pedatisectae rhizoma samples were shown in Table 7. The molecular ion peaks of Pinelliae pedatisectae rhizoma and triglochinic acid were the same (187), which is consistent with the mass of triglochinic acid ion $\left(\mathrm{C}_{7} \mathrm{H}_{7} \mathrm{O}_{6}{ }^{-}\right)$. The molecular ion peaks were further confirmed by MS/MS, showing that the secondary ions were the same: 143 and 99.

According to the results of HPLC-MS, the peak retention time detected in Pinelliae pedatisectae rhizoma was consistent with that of triglochinic acid. Furthermore, the mass-to-charge ratio of the selected two pairs of daughter ions were consistent. The relative abundance of qualitative ions of Pinelliae pedatisectae rhizoma and triglochinic acid were within the range of tolerance $( \pm 20 \%),{ }^{\mathbf{1 4 , 1 5}}$ while their retention times were also consistent. Since the triglochinic acid peak was not detected in Pinelliae rhizoma, it can be verified that Pinelliae rhizoma does not contain triglochinic acid, while Pinelliae pedatisectae rhizoma does.

\section{Discussion}

Pinelliae pedatisectae rhizoma and Pinelliae rhizoma belong to the same genus, and share similar characteristics. The processed or young tubers of Pinellia pedatisecta are sold as Pinelliae rhizoma in herbal medicine market. While the identification has always been difficult to achieve. In addition, Pinelliae pedatisectae rhizoma in large size consists of the main tuber and a few small attached ones, resembling a tiger's claw. ${ }^{16}$ Due to the factors like provenance, soil, pests and diseases, and cultivation, some trait variations occur to Pinelliae rhizoma as well: one or several small tubers appear around the major tuber, which is similar to the characters of Pinelliae pedatisectae rhizoma and is easily misidentified as Pinelliae pedatisectae rhizoma. Pinelliae rhizoma, with its various forms of prepared drug in pieces, is popular in clinical use. On the market, the products of Pinelliae pedatisectae rhizoma are sold as the counterfeit of Pinelliae rhizoma because after processing, their color and surface characteristics further change. Especially after slicing, Pinelliae rhizoma and Pinelliae pedatisectae rhizoma are almost indistinguishable from their appearances.

The chemical constituents of Pinelliae rhizoma and Pinelliae pedatisectae rhizoma are similar. Pinelliae pedatisectae rhizoma contains a variety of alkaloids, dipeptides, amino acids, organic acids, nucleosides, and polysaccharides, ${ }^{\mathbf{1 7 - 2 0}}$ the same does Pinelliae rhizoma except for dipeptides. ${ }^{21-27}$ At present, morphological identification, thin layer chromatography identification and fingerprint identification are the main identification methods for Pinelliae rhizoma. However, morphological identification faces great difficulty especially after processing or slicing. Chen et al. ${ }^{28}$ identified one more spot in Pinelliae rhizoma which was not detected in Pinelliae pedatisectae rhizoma by means of thin-layer chromatography, but this method could not determine whether Pinelliae rhizoma was incorporated or not. $\mathrm{Lu}^{9}$ established the fingerprints of Pinelliae rhizoma and Pinelliae pedatisectae rhizoma, in which Pinelliae rhizoma had three more chromatographic peaks than Pinelliae pedatisectae rhizoma, but it was also unable to identify the incorporated Pinelliae rhizoma.

Triglochinin, which can be hydrolyzed into triglochinic acid, was found in the flowers of Triglochin maritima, ${ }^{29}$ the young leaves of Alocasia ${ }^{30,31}$ and Ranunculaceae genus. ${ }^{32}$ The Alocasia genus is toxic, and the whole plant contains cyanogenic glycoside. However, whether cyanogenic glycoside is the main substance causing the toxicity of Alocasia macrorrhizos is not fully understood. According to the literatures, cyanogenic glycoside itself is not toxic, but it can be degraded by $\beta$-glucosidase and $\alpha$-hydroxynitrile lyase, thereby releasing the toxic hydrogen cyanide (HCN) as well as glucose and aldehydes or ketones, ${ }^{33}$ resulting in toxic effects. The triglochinic acid was found in Pinelliae pedatisectae rhizoma, but whether it is toxic remains to be confirmed in future research. Pinelliae rhizoma and Pinelliae pedatisectae rhizoma are of the same genus Araceae, which is inherently toxic: mainly stimulating toxic effects, caused by the shared raphides and lectin proteins. ${ }^{34}$ Furthermore, their efficacy and clinical applications are different. Therefore, the use should be strictly differentiated, and the safety of Pinelliae rhizoma mixed with Pinelliae pedatisectae rhizoma should be concerned as well.

In addition, the history of artificial cultivation of Pinellia ternata is short. The seeds of Pinellia ternata are mostly wild, 
sometimes being mixed with that of Pinellia pedatisecta, resulting in a small amount of Pinelliae pedatisectae rhizoma identified in Pinelliae rhizoma. Now it is highly necessary to strengthen the research on the seeds standard of Pinellia ternata, and control its quality from the source.

In this study, for the first time we isolated the triglochinic acid from Pinelliae pedatisectae rhizoma and established the HPLC identification method and LC-MS verification method. This method is stable, accurate, and widely applicable. It can be used for the identification of Pinelliae pedatisectae rhizoma in Pinelliae rhizoma materials or prepared drugs. As a supplement to the quality control of Pinelliae rhizoma in the Chinese Pharmacopoeia, this method effectively combats the situation of adulteration and counterfeiting in the market, protecting the interests of farmers, planting enterprises, merchants, and companies. It also promotes the quality control of Pinelliae rhizoma, effectively ensuring the safety of clinical use.

\section{Conflicts of interest}

There are no conflicts to declare.

\section{Acknowledgements}

The authors greatly appreciate financial support from the State Administration of Traditional Chinese Medicine of China (ZYBZH-Y-SC-40), Sichuan Provincial Department of Education (2050205, 2016NFP0076 and 17CZ0013), Science and Technology Department of Sichuan Province (16ZHSF0451 and 17KJFP0230), National Foundation for Training Basic Science Talents (J1310034-04).

\section{References}

1 Chinese Pharmacopoeia Commission, Pharmacopoeia of the People's Republic of China, The Medicine Science and Technology Press of China, Beijing, 2015.

2 P. G. Xiao, Modern Chinese Materia Medica, Chemical Industry Press, Beijing, 2002.

3 Shandong Food and Drug Administration, Standard of traditional Chinese medicine in Shandong province, Shandong Science and Technology Press, Jinan, 2012.

4 Hubei Food and Drug Administration, Standard of traditional Chinese medicine in Hubei province, Hubei Science and Technology Press, Wuhan, 2009.

5 Jiangsu Food and Drug Administration, Standard of traditional Chinese medicine in Jiangsu province, Phoenix Science Press, Nanjing, 2016.

6 Nanjing University of Traditional Chinese Medicine, Dictionary of Traditional Chinese Medicine, Shanghai Science and Technology Press, Shanghai, 2006.

7 Chinese Botany Editorial Committee and Chinese Academy of Sciences, Chinese Flora, Science Press, Beijing, 2004.

8 Chinese Patent Medicine Prescription Database, https:// db.yaozh.com/chufang, accessed March 2019.
9 Traditional Chinese Medicine Prescription Database, https:// db.yaozh.com/fangji, accessed March 2019.

$10 \mathrm{~J}$. W. Li, R. C. Xu and P. F. Wang, Asia Pacific Journal of traditional Chinese Medicine, 2015, 11(24), 23-26.

11 D. H. Lu, master's thesis, Chengdu University of Traditional Chinese Medicine, 2012.

$12 \mathrm{H}$. Wu, W. Li and W. K. Zhang, China J. Chin. Mater. Med., 2003, 28(9), 836-839.

13 R. Eyjólfsson, Phytochemistry, 1970, 9(4), 845-851.

14 Standardization Administration of the People's Republic of China, National Standards of People's Republic of China(GB/ $T$ 6379.2), Standards Press of China, Beijing, 2004.

15 Standardization Administration of the People's Republic of China, National Standards of People's Republic of China(GB/ T 24800.2), Standards Press of China, Beijing, 2009.

16 D. Lu, Y. M. Chi and Y. Q. Zhao, Chin. Tradit. Pat. Med., 2013, 35(06), 1274-1278.

$17 \mathrm{~W}$. Man, master's thesis, Changchun University of Traditional Chinese Medicine, 2012.

18 R. B. Wang, C. Q. Wang and X. L. Liu, Journal of Changchun University of Traditional Chinese Medicine, 2010, 26(04), 590592.

19 L. Ju, Y. M. Chi and T. H. Wang, Asia Pacific Journal of Traditional Chinese Medicine, 2016, 12(09), 30-32.

20 X. L. Mo, Y. J. Liu and T. Li, Pharmacy and Clinics of Chinese Materia Medica, 2017, 8(06), 13-16.

$21 \mathrm{~W}$. B. $\mathrm{Hu}, \mathrm{H}$. Wang and S. F. Zhang, China Resources Comprehensive Utilization, 2016, 34(10), 57-59.

22 Y. H. Liu, J. H. Guo and W. T. Liu, Journal of Northwest A\&F University (Natural Science Edition), 2015, 43(09), 171-177.

23 J. K. Xu, T. L. Zhang and G. Q. Yi, J. Shenyang Pharm. Univ., 2010, 27(06), 429-433.

24 J. Xiao, B. K. Huang and G. W. Wang, Fitoterapia, 2014, 93(04), 1-17.

25 Z. H. Zhang, Z. Dai and R. Xiao, J. Chin. Med. Mater., 2013, 36(10), 1620-1622.

26 P. Z. Wang, B. X. Du and Y. P. Fu, China J. Tradit. Chin. Med. Pharm., 2018, 33(03), 911-914.

27 H. L. Xu, J. P. Chang and Z. S. Liang, Chinese Traditional Medicine, 2016, 38(9), 1998-2002.

28 Y. Chen, Y. Q. Qiu and S. W. Liang, Asia-Pacific Traditional Medicine, 2018, 14(02), 44-46.

29 Y. M. Zhu, Inner Mongolia Plant Medicine, Inner Mongolia People's Publishing House, Inner Mongolia, 2000.

30 J. W. Huang, G. F. Huang and Y. Q. Lv, Strait Pharmaceutical Journal, 2014, 26(08), 19-20.

31 X. Lei, Z. H. Xu and Y. Wang, Chin. J. New Drugs Clin. Rem., 2013, 32(03), 163-166.

32 W. Zheng, master's thesis, Zhejiang University, 2006.

33 Y. Zhang, D. Q. Tang and M. B. Zhou, Biotechnol. Bull., 2009, (04), 12-15.

34 F. G. Zhu, master's thesis, Nanjing University of Traditional Chinese Medicine, 2012. 